\title{
Pseudo one-compartment models. Methods for assessing the peripheral compartment for them
}

\author{
N. Asmanova*, A.I. Ilin \\ JSC "Scientific center for anti-infection drugs", Almaty, Kazakhstan \\ *e-mail: asmanova@inbox.ru
}

Key words: pseudo one-compartment model, first order absorption, direct and inverse problems in pharmacokinetics

Motivation and aim: If $k_{a}=k_{21}$, then the equation of PK curve for two-compartment model with first order absorption (2ev) is transformed from eq. (1) into (2). In solving inverse problems, it is identified only as one-compartment model-lev (3), where $k_{a}>k_{10}$ $[1,2]$ :

$C_{1}=A_{1} e^{-\alpha t}+A_{2} e^{-\beta t}-\left(A_{1}+A_{2}\right) e^{-k_{a} t}$

$C_{1}=A_{2}\left(e^{-\beta t}-e^{-\alpha t}\right)$

$C_{1}=A\left(e^{-k_{10} t}-e^{-k_{a} t}\right)$

So, actual loss of the term $-\left(A_{1}+A_{2}\right) e^{-k_{a} t}$, associated with absorption, turns into a fictitious disappearance of the distribution phase. The reason for this problem is the ambiguous role of the volume of distribution of the drug, in practice of pharmacokinetics it is not measured, but is calculated.

Methods: The analysis of eqs. (1-3) and the solution of direct and inverse problems for them.

Results: It is shown that an approximate evaluation of the peripheral compartment can be obtained with the help of the parameters of the inversion line [1], relation (4) and their comparison with intravascular (iv) bolus data (5). Here $A U C_{1}$ and $A U C_{2}$ are the areas under the PK curves of the central and peripheral compartments, $k_{a}$ and $k_{10}$ are absorption and elimination rate constants respectively.

$$
\begin{aligned}
& A U C_{2\left(k_{a}<k_{10}\right)}>A U C_{2\left(k_{a}=k_{10}\right)}>A U C_{2\left(k_{a}>k_{10}\right)} \\
& \left.\left.A U C_{2} / A U C_{1}\right)_{e v} \approx A U C_{2} / A U C_{1}\right)_{i v}
\end{aligned}
$$

Conclusion: The inverse problem for eq. (2) is solvable only for a known volume, but there are no methods for determining it. Equation (3) is its pseudo one-compartment version, so the calculation of the drug dosage regimen on its basis is incorrect.

\section{References}

1. Asmanova N., Ilin A.I. (2016) Futures of solution of some inverse problems in PK models with first order absorption. Abstracts: International Conference Mathematical modeling and High Performance Computing in Bioinformatics, Biomedicine and biotechnology MM-HPC-BBB-2016, Novosibirsk, Russia, 29 August - 2 September, p. 15.

2. Asmanova N., Ilin A.I. (2016) Flip-flop phenomenon in two-compartment model with first order absorption, ibid, p. 16. 